IFUP-TH 32/97

\title{
Group theoretical derivation of Liouville action for Regge surfaces t.
}

\author{
Pietro Menotti \\ Dipartimento di Fisica dell'Università, Pisa 56100, Italy and \\ INFN, Sezione di Pisa
}

July 1997

${ }^{1}$ This work is supported in part by M.U.R.S.T. 


\begin{abstract}
We show that the structure of the Liouville action on a two dimensional Regge surface of the topology of the sphere and of the torus is determined by the invariance under the transformations induced by the conformal Killing vector fields and under modular transformations.
\end{abstract}

\title{
1 Introduction
}

The Liouville action plays a key role in two dimensional gravity. It provides the quantum theory with a highly non trivial dynamics, while the corresponding classical theory is completely trivial.

On the continuum the Liouville action has been derived in [1] and it is a non perturbative result. On a two dimensional Regge surface, i.e. on a surface which is everywhere flat except at isolated points, the exact expression of the Liouville action has been derived in [2]. The interest in considering Regge surfaces is that they provide the reduction of the functional integral to a finite number of degrees of freedom with a simple geometrical meaning [3]. The derivation [2], employs the $Z$-function regularization of functional determinants on singular Riemann surfaces and the Riemann-Roch theorem to fix the correct self-adjoint extension of the conformal Lichnerowich-De Rahm operator.

One wonders whether a simpler argument can explain the structure of the action. What we shall show here is that, under reasonable assumptions that we shall state in the following, the structure of the Liouville action for a Regge surface is dictated by group theory alone, i.e. by the invariance of the action under the transformations induced by the conformal Killing vector fields and under modular transformations.

\section{Structure of the discretized Liouville action}

The Liouville action in Polyakov covariant non local form is given by 


$$
S_{l}=\frac{26}{96 \pi}\left\{\int d^{2} \omega d^{2} \omega^{\prime}(\sqrt{g} R)_{\omega} \frac{1}{\square}\left(\omega, \omega^{\prime}\right)(\sqrt{g} R)_{\omega^{\prime}}-2\left(\log \frac{A}{A_{0}}\right) \int d^{2} \omega \sqrt{g} R\right\}
$$

where in the case of spherical topology

$$
\frac{1}{\square}\left(\omega, \omega^{\prime}\right)=\frac{1}{2 \pi} \log \left|\omega-\omega^{\prime}\right|
$$

and $A$ is the area of the surface. $A_{0}$ is the reference area i.e. the value of the area due to the $\sigma_{0}$ in the following decomposition of the conformal factor $\sigma$

$$
\sigma(\omega)=\sigma_{0}(\omega)+\lambda_{0}, \quad \sigma_{0} \approx-2 \log |\omega| \text { for } \omega \rightarrow \infty
$$

The last term in eq.(11) in important as it provides the correct transformation properties of the action under uniform rescaling [四].

In [2] the Liouville action has been derived for a two dimensional Regge surface, i.e. a surface which is everywhere flat except at isolated points where conical singularities are present; the result for spherical topology was

$$
S_{l}=\frac{26}{12}\left\{\sum_{i, j \neq i} \frac{\left(1-\alpha_{i}\right)\left(1-\alpha_{j}\right)}{\alpha_{i}} \log \left|w_{i}-w_{j}\right|+\lambda_{0} \sum_{i}\left(\alpha_{i}-\frac{1}{\alpha_{i}}\right)-\sum_{i} F\left(\alpha_{i}\right)\right\}
$$

where $\alpha_{i}$ are the apertures of the conical singularities ( $\alpha=1$ is the plane), $\omega_{i}$ the locations of the singularities and $\lambda_{0}$ the scale parameter occurring in the Regge conformal factor (see eq.(5) below). $F(\alpha)$ is a smooth function for which an integral representation can be given. A similar formula can be derived for the torus topology (see [2] and eq.(47) below). The idea of using the complex plane with conical singularities to describe a Regge geometry is found in [5] [6], while the derivation of eq.(41) was given in [2] by extending a technique developed in [6]. We recall that the description by means of the complex plane is completely equivalent to the one in terms of the bone lengths, but the former in two dimensions looks more powerful.

We shall start from the topology of the sphere. As usual we shall describe the surface with the topology of a sphere by a single chart given by the complex plane completed 
by the point at infinity (Riemann sphere) and the conformal factor describing the Regge geometry is given by [5], [6], [2]

$$
e^{2 \sigma}=e^{2 \lambda_{0}} \prod_{i=1}^{N}\left|\omega-\omega_{i}\right|^{2\left(\alpha_{i}-1\right)}, \quad \sigma \equiv \sigma\left(\omega ; \lambda_{0}, \omega_{i}, \alpha_{i}\right)=\lambda_{0}+\sum_{i}\left(\alpha_{i}-1\right) \log \left|\omega-\omega_{i}\right|
$$

with the restriction $\sum_{i=1}^{N}\left(1-\alpha_{i}\right)=2$, i.e. the sum of the deficits must be equal to the Euler characteristic. Due to the presence of six conformal Killing vector fields for the sphere topology such a conformal factor is unique [7] up to an $S L(2, C)$ transformation. Under

$$
\omega^{\prime}=\frac{\omega a+b}{\omega c+d}, \quad \omega=\frac{\omega^{\prime} d-b}{-\omega^{\prime} c+a}, \quad a d-b c=1
$$

the new conformal factor is given by

$$
\sigma^{\prime}\left(\omega^{\prime} ; \lambda_{0}, \omega_{i}, \alpha_{i}\right)=\sigma\left(\omega^{\prime} ; \lambda_{0}^{\prime}, \omega_{i}^{\prime}, \alpha_{i}^{\prime}\right)
$$

with

$$
\lambda_{0}^{\prime}=\lambda_{0}+\sum_{i=1}^{N}\left(\alpha_{i}-1\right) \log \left|\omega_{i} c+d\right|, \quad \omega_{i}^{\prime}=\frac{a \omega_{i}+b}{c \omega_{i}+d}, \quad \alpha_{i}^{\prime}=\alpha_{i} .
$$

The area $A$

$$
A=e^{2 \lambda_{0}} \int d^{2} \omega\left|\omega-\omega_{i}\right|^{2\left(\alpha_{i}-1\right)}
$$

being a geometric invariant is left unchanged.

We now must construct a discrete transcription of the non local action (1) in terms of the $3 N$ parameters $\omega_{1 x}, \omega_{1 y}, \ldots \omega_{N x}, \omega_{N y}, \alpha_{1} \ldots \alpha_{N-1}, \lambda_{0}$ characterizing the Regge conformal factor. The Green function on the plane is

$$
\frac{1}{2 \pi} \log \left|\omega_{i}-\omega_{j}\right|
$$

and the curvature is concentrated at the points $\omega_{i}$ and characterized by $\alpha_{i}$. As the integrated curvature at the singularity $\omega_{i}$ is given by $4 \pi\left(1-\alpha_{i}\right)$ it would be natural to consider for the action the combination $\left(1-\alpha_{i}\right)\left(1-\alpha_{j}\right) \log \left|\omega_{i}-\omega_{j}\right|$. However, while providing the right behavior for small angular deficit, i.e. small $1-\alpha_{i}$, it is easily checked 
(and it will be proved in the following) that the ensuing structure is not correct as it is not invariant under $S L(2, C)$. Thus we must consider the more general structure

$$
\sum_{i, j} K_{i j}[\alpha] \log \left|\omega_{i}-\omega_{j}\right|+B\left(\lambda_{0}, \alpha\right)
$$

where $K_{i j}[\alpha]$ has the following properties

$$
K_{i j}[\alpha]=K_{j i}[\alpha], \quad K_{i i}[\alpha]=0 .
$$

In fact $K$ has to be a symmetric function of $i, j$ which depends only on the (singular) curvatures. $B$ is a function to be determined of the $\alpha$ 's and the scale parameter $\lambda_{0}$. Under the $S L(2, C)$ transformation (8) we have

$$
\left|\omega_{i}^{\prime}-\omega_{j}^{\prime}\right|=\frac{\left|\omega_{i}-\omega_{j}\right|}{\left|c \omega_{i}+d\right|\left|c \omega_{j}+d\right|} .
$$

Invariance of the action under such a transformation gives us

$$
-2 \sum_{i, j} K_{i j}[\alpha] \log \left|c \omega_{i}+d\right|+B\left(\lambda_{0}^{\prime}, \alpha\right)=B\left(\lambda_{0}, \alpha\right)
$$

Let us consider first a dilatation i.e. a transformation with $a=1 / d=\Lambda, b=c=0$. We have

$$
2 \sum_{i, j \neq i} K_{i j}[\alpha] \log \Lambda+B\left(\lambda_{0}+2 \log \Lambda, \alpha\right)=B\left(\lambda_{0}, \alpha\right)
$$

The above relation tells us that $B$ is a linear function of $\lambda_{0}$ i.e.

$$
B\left(\lambda_{0}, \alpha\right)=-\lambda_{0} \sum_{i, j} K_{i j}[\alpha]+F[\alpha]
$$

Thus we have reached the structure

$$
\sum_{i, j} K_{i j}[\alpha] \log \left|\omega_{i}-\omega_{j}\right|-\lambda_{0} \sum_{i, j} K_{i j}[\alpha]+F[\alpha]
$$

We impose now the invariance of the above action under the general transformation (6)

$$
0=2 \sum_{i, j} K_{i j}[\alpha] \log \left|c \omega_{i}+d\right|+\sum_{i}\left(\alpha_{i}-1\right) \log \left|c \omega_{i}+d\right| \sum_{m, n} K_{m n}[\alpha]
$$


It is useful to define $K_{i j}[\alpha]=\left(1-\alpha_{i}\right)\left(1-\alpha_{j}\right) H_{i j}[\alpha]$ and, as the $\omega_{i}$ are independent variables we obtain

$$
2 \sum_{j} H_{i j}[\alpha]\left(1-\alpha_{j}\right)=\sum_{m, n}\left(1-\alpha_{m}\right)\left(1-\alpha_{n}\right) H_{m n}[\alpha]
$$

Notice that the second member does not depend on $i$. We now specify that $K_{i j}[\alpha]$ depends on $\alpha_{i}$ and $\alpha_{j}$ i.e. only on the (singular) curvatures at $\omega_{i}$ and $\omega_{j}$. This means that $H_{i j}[\alpha]=h\left(\alpha_{i}, \alpha_{j}\right)$. Then eq.(19) becomes

$$
2 \sum_{j} h\left(\alpha_{i}, \alpha_{j}\right)\left(1-\alpha_{j}\right)=\sum_{m, n}\left(1-\alpha_{m}\right)\left(1-\alpha_{n}\right) h\left(\alpha_{m}, \alpha_{n}\right) .
$$

The above equation must hold for any choice of $\alpha_{i}>0$ respecting the Euler relation $\sum_{i}\left(1-\alpha_{i}\right)=2$ and for any number $N$ of vertices. Given $\alpha_{1}$ and $\alpha_{2}$ at will we shall choose all the other $\alpha$ 's equal and thus given by

$$
1-\bar{\alpha}=\frac{\alpha_{1}+\alpha_{2}}{N-2}
$$

Substituting into (20) we have for $i=1$

$$
\begin{gathered}
\alpha_{1}\left(1-\alpha_{2}\right) h\left(\alpha_{1}, \alpha_{2}\right)+\alpha_{1}\left(\alpha_{1}+\alpha_{2}\right) h\left(\alpha_{1}, \bar{\alpha}\right)= \\
\left(1-\alpha_{2}\right)\left(\alpha_{1}+\alpha_{2}\right) h\left(\alpha_{2}, \bar{\alpha}\right)+\frac{(N-3)}{2(N-2)}\left(\alpha_{1}+\alpha_{2}\right)^{2} h(\bar{\alpha}, \bar{\alpha}) .
\end{gathered}
$$

The above equation has to hold for any $N$ and thus also in the limit $N \rightarrow \infty$ i.e.

$\alpha_{1}\left(1-\alpha_{2}\right) h\left(\alpha_{1}, \alpha_{2}\right)+\alpha_{1}\left(\alpha_{1}+\alpha_{2}\right) h\left(\alpha_{1}, 1\right)=\left(1-\alpha_{2}\right)\left(\alpha_{1}+\alpha_{2}\right) h\left(\alpha_{2}, 1\right)+\frac{\left(\alpha_{1}+\alpha_{2}\right)^{2}}{2} h(1,1)$.

The equation is homogeneous in $h$ and so we shall not be able, using simply group theory to fix the overall proportionality constant. We shall normalize $h$ to $h(1,1)=2$. A solution to eq.(23) (and also to eq.(22)) is given by

$$
h\left(\alpha_{1}, \alpha_{2}\right)=\frac{1}{\alpha_{1}}+\frac{1}{\alpha_{2}} .
$$

We shall put

$$
h\left(\alpha_{1}, \alpha_{2}\right)=\frac{1}{\alpha_{1}}+\frac{1}{\alpha_{2}}+r\left(\alpha_{1}, \alpha_{2}\right)
$$


with obviously $r(1,1)=0$, and our aim is to prove that $r\left(\alpha_{1}, \alpha_{2}\right)$ is identically zero. The equation for $r\left(\alpha_{1}, \alpha_{2}\right)$ now is

$$
\alpha_{1}\left(1-\alpha_{2}\right) r\left(\alpha_{1}, \alpha_{2}\right)+\alpha_{1}\left(\alpha_{1}+\alpha_{2}\right) r\left(\alpha_{1}, 1\right)=\left(1-\alpha_{2}\right)\left(\alpha_{1}+\alpha_{2}\right) r\left(\alpha_{2}, 1\right)
$$

Putting in eq.(26) $\alpha_{2}=1$ we have

$$
\alpha_{1}\left(\alpha_{1}+1\right) r\left(\alpha_{1}, 1\right)=0
$$

from which $r\left(\alpha_{1}, 1\right)=0$ and substituting into eq.(26) we get finally $r\left(\alpha_{1}, \alpha_{2}\right)=0$.

With regard to the function $F[\alpha]$ appearing in eq.(17), being the $\alpha$ 's invariants under $S L(2, C)$ transformations, nothing can be said from group theory alone; the only thing we notice here is that as it happens for the exact discretized action (雨) for small angular deficits, i.e. near the continuum limit it becomes

$$
F[\alpha] \approx F[1]+\frac{\partial F}{\partial \alpha_{1}}[1] \sum_{i}\left(\alpha_{i}-1\right)=F[1]-\chi \frac{\partial F}{\partial \alpha_{1}}[1]
$$

i.e. a topological invariant.

Once the non perturbative structure in known, the overall coefficient $26 / 24$ can be borrowed from perturbation theory as was originally done by Polyakov [1]. We recall however that the procedures [1] and [2] provide also the correct normalization.

In conclusion for the sphere topology we have reproduced eq.(任) except for the last term which in both formulae becomes a topological invariant in the continuum limit.

For the torus the invariance group is confined to the modular group times the translations.

The conformal factor describing a Regge surface with the topology of the torus is given by [2]

$$
\sigma(\omega)=\lambda_{0}+2 \pi \sum_{i=1}^{N}\left(\alpha_{i}-1\right) G\left(\omega-\omega_{i} \mid \tau\right)
$$

where $\omega=x+\tau y$, being the fundamental region given by the square $0 \leq x<1,0 \leq y<1$ and $G\left(\omega-\omega^{\prime} \mid \tau\right)$ is the Green function on the torus [8]

$$
\begin{gathered}
\square G\left(\omega-\omega^{\prime} \mid \tau\right)=\delta^{2}\left(\omega-\omega^{\prime}\right)-\frac{1}{\tau_{2}} \\
G\left(\omega-\omega^{\prime} \mid \tau\right)=\frac{1}{2 \pi} \log \left|\frac{\vartheta_{1}\left(\omega-\omega^{\prime} \mid \tau\right)}{\eta(\tau)}\right|-\frac{\left(\omega_{y}-\omega_{y}^{\prime}\right)^{2}}{2 \tau_{2}} .
\end{gathered}
$$


$\vartheta_{1}(\omega \mid \tau)$ is the Jacobi $\vartheta$-function and

$$
\eta(\tau)=e^{\frac{i \pi \tau}{12}} \prod_{n=1}^{\infty}\left[1-e^{2 i n \pi \tau}\right]
$$

The discrete structure inherited from (11) is

$$
2 \pi \sum_{i, j} K_{i j}[\alpha, \tau] G\left(\omega_{i}-\omega_{j} \mid \tau\right)+B\left(\lambda_{0}, \alpha, \tau\right)
$$

We recall that $G\left(\omega_{1}-\omega_{2} \mid \tau\right)$ is modular invariant i.e. under the modular transformation

$$
\tau \longrightarrow \tau^{\prime}=\frac{\tau a+b}{\tau c+d}
$$

with $(a, b, c, d) \in \mathbf{Z}$ and $a d-b c=1$ and

$$
\omega^{\prime}=\frac{\omega}{\tau c+d}
$$

we have

$$
G\left(\omega_{1}-\omega_{2} \mid \tau\right)=G\left(\omega_{1}^{\prime}-\omega_{2}^{\prime} \mid \tau^{\prime}\right)
$$

We shall assume now that at short distances the divergence behavior of the action should be independent of the topology of the manifold i.e. for $\omega_{1}-\omega_{2} \rightarrow 0$ the discrete Liouville action should diverge like on the sphere which, as

$$
2 \pi G\left(\omega_{1}-\omega_{2} \mid \tau\right) \approx \log \left|\omega_{1}-\omega_{2}\right|
$$

imposes again

$$
K_{12}[\alpha]=\left(1-\alpha_{1}\right)\left(1-\alpha_{2}\right)\left(\frac{1}{\alpha_{1}}+\frac{1}{\alpha_{2}}\right) .
$$

We must now determine the function $B\left(\lambda_{0}, \alpha, \tau\right)$. Under the modular transformation (34) and (35) $\lambda_{0}$ goes over to

$$
\lambda_{0}^{\prime}=\lambda_{0}+\log |\tau c+d| .
$$

Due to the modular invariance of the Green function we must have

$$
B\left(\lambda_{0}, \alpha, \tau\right)=B\left(\lambda_{0}^{\prime}, \alpha, \tau^{\prime}\right)=B\left(\lambda_{0}+\log |\tau c+d|, \alpha, \frac{\tau a+b}{\tau c+d}\right) .
$$


If we write, without any loss of generality

$$
B\left(\lambda_{0}, \alpha, \tau\right)=C\left(\lambda_{0}-\log \left|2 \pi \eta^{2}(\tau)\right|, \alpha, \tau\right)
$$

recalling that $\eta^{24}(\tau)$ is a modular cusp form of weight 12 i.e.

$$
\eta^{24}\left(\frac{\tau a+b}{\tau c+d}\right)=(c \tau+d)^{12} \eta^{24}(\tau)
$$

we have that $C$ is invariant under modular transformations i.e.

$$
C(x, \alpha, \tau)=C\left(x, \alpha, \frac{\tau a+b}{\tau c+d}\right) .
$$

In order to specialize further the function $C$ let us consider the case when all $\omega_{i}$ are all close together. In this case the geometry is given by a local deformation with zero integrated curvature of a flat torus of dimensions much larger than the size of the deformation. In the described situation and $\omega$ near the $\omega_{i}$ 's the conformal factor (29) becomes

$$
\sigma(\omega) \approx \lambda_{0}+\sum_{i}\left(\alpha_{i}-1\right) \log \left|\omega-\omega_{i}\right|
$$

and as it is easily checked the conformal factors (44) and the following (45)

$$
\sigma^{\prime}\left(\omega^{\prime}\right) \approx \lambda_{0}-\log k+\sum_{i}\left(\alpha_{i}-1\right) \log \left|\omega^{\prime}-k \omega_{i}\right|
$$

describe the same geometry. Imposing in such a limit the invariance of the action (33) under $\omega_{i} \rightarrow k \omega_{i} \rightarrow$ and $\lambda_{0} \rightarrow \lambda_{0}-\log k$ we obtain, taking into account that for the torus $\sum_{i}\left(1-\alpha_{i}\right)=0$

$$
B=2\left(\lambda_{0}-\log \left|2 \pi \eta^{2}\right|\right) \sum_{i}\left(\alpha_{i}-\frac{1}{\alpha_{i}}\right)+2 F[\alpha, \tau]
$$

with $F[\alpha, \tau]$ invariant under modular transformations. Obviously any $F[\alpha, \tau]$, even discontinuous in $\tau$, provided it is invariant under (34) will satisfy our invariance requirements. It is however of interest to see what happens if for $e^{S_{l}}$ we remain in the domain of modular functions. Any modular invariant function can be written as a rational function of the modular invariant $j(\tau)$ [9]. Moreover $j(\tau)$ has a simple pole at infinity and provides a 
bijection between $H / G$ onto the complex plane $C$, where $H / G$ the quotient of the upper complex plane with the modular group [9]. Thus if we want to avoid $\operatorname{singularities~in~} \tau$ we must choose $F$ independent of $\tau$. With such a choice we have for the torus

$$
S_{l}=\frac{26}{12}\left[\sum_{i, j \neq i} \frac{\left(1-\alpha_{i}\right)\left(1-\alpha_{j}\right)}{\alpha_{i}} 2 \pi G\left(\omega_{i}-\omega_{j} \mid \tau\right)+\left(\lambda_{0}-\log \left|2 \pi \eta^{2}\right|\right) \sum_{i}\left(\alpha_{i}-\frac{1}{\alpha_{i}}\right)+F[\alpha]\right] .
$$

where again the normalization has been borrowed from perturbation theory. Eq.(47) is identical to the one derived in [2] except for the last term $F[\alpha]$ which here is left generic while in [2] is completely determined. However both terms in the continuum limit go over to a topological invariant.

\section{Conclusions}

The complete determination of the Liouville action for a Regge surface in two dimensions has been given in [2] both for the sphere topology and the torus topology and it was shown that, as expected, the obtained actions are invariant under the continuous transformations induced by the conformal Killing vector fields and under the modular group. Here the question has been addressed of how far such symmetries restrict the translation of the continuum Polyakov covariant non local expression of the Liouville action to the discrete case.

Is is found that, under reasonable assumptions, the action for the sphere and torus topology are completely fixed except for a function of the deficit angles which in the continuum goes over to a topological invariant and the overall normalization of the action which has to be borrowed e.g. from perturbation theory.

Thus even if the present derivation does not replace the full one given in [2], it provides a simpler treatment and shows that the invariance principles are practically sufficient to fix the form of the action. 


\section{References}

[1] A.M. Polyakov, Phys. Lett. 103B (1984) 207; J. Polchinski, Comm. Math. Phys. 104 (1986) 37; O. Alvarez, Nucl. Phys. B216 (1983) 125; G. Moore, P. Nelson, Nucl. Phys. B266 (1986) 58.

[2] P. Menotti, P.P. Peirano Phys. Lett. 353B (1995) 444; Nucl.Phys. B 473 (1996) 426.

[3] P. Menotti, P.P. Peirano "Functional integration for Regge gravity" IFUP-TH-3-97, Talk given at 2nd Meeting on Constrained Dynamics and Quantum Gravity, Santa Margherita Ligure, Italy, 17-21 Sep. 1996, gr-qc/9702020.

[4] M.J. Duff Class. Quantum Gravity 11 (1994) 1387; J.S. Dowker Class. Quantum Gravity 11 (1994) L7.

[5] D. Foerster, Nucl. Phys. B283 (1987) 669; Nucl. Phys. B291 (1987) 813

[6] E. Aurell, P. Salomonson, Comm. Math. Phys. 165 (1994) 233; Further results on Functional Determinants of Laplacians in Simplicial Complexes, (hep-th/9405140).

[7] P. Ginsparg, Les Houches, Session XLIX, (1988), Elsevier Science Publishers (1989).

[8] P. Di Francesco, H. Saleur, J.D. Zuber, Nucl. Phys. B290 (1987) 527; E. Guadagnini, M. Martellini, M. Mintchev J. Math. Phys. 31 (1990) 1226.

[9] J.-P. Serre, A course in arithmetics, Springer Verlag, New York, 1973. 